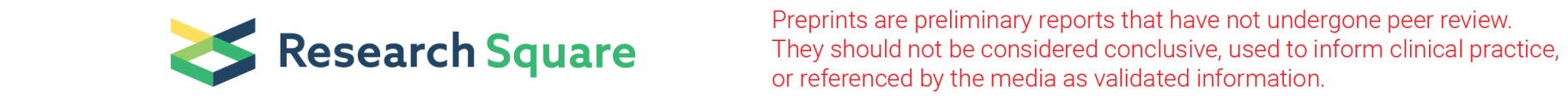

\title{
Geriatricians' Standpoint Regarding the Informed Consent Process among Patients with Alzheimer's Disease: The Lebanese Context
}

Mohammed Ali Fakhro ( $\square$ m.fakhro@lgu.edu.lb)

Lebanese German University

\section{Research article}

Keywords: Autonomy, Informed Consent, Decision-making Capacity, Alzheimer's Disease

Posted Date: December 5th, 2019

DOI: https://doi.org/10.21203/rs.2.18271/v1

License: (c) (i) This work is licensed under a Creative Commons Attribution 4.0 International License. Read Full License 


\section{Abstract}

Background Alzheimer's disease (AD) is a degenerative brain disease and the most common cause of dementia among older adults. Moreover, as the disease progress, patients lose their capacity to make important decisions as related to their wishes. The primary objective was to characterize the Lebanese Geriatricians' standpoint regarding the decision-making capacity (DMC) to consent to research and/or treatment participation among their patients with Alzheimer Disease (AD). The secondary objective was to propose an algorithm for a reliable, comprehensive, and valid assessment of the DMC.

Methods A cross-sectional descriptive study was designed, where a list of active members of the Lebanese Society for Geriatric Medicine was obtained, and participants were contacted via a phone call, to explain the study purpose, and send them thereafter the survey link.

Results Nine Geriatricians with a mean of $14.7 \pm 9.6$ years of experience completed the survey. Moreover, $66.67 \%$ of the Geriatricians failed to recognize the need to confirm the patient's capacity to make decisions as related to her/his own wishes as related to the process of informed consent (IC). Despite that, $88.9 \%$ of them stated that they tested for DMC among their patients with AD.

Conclusion This study finding revealed that Lebanese Geriatricians had a poor understanding of both the concept of autonomy and the criteria needed for the IC process. Moreover, they were unable to select adequate tools to assess and judge the DMC.

\section{Background}

According to the Population Reference Bureau's 2018 population data sheet, the global fertility rate was declining for the past few decades, yet remaining high enough to allow for continual population growth. This fact combined with the increase in life expectancy is causing the global population to age rapidly ${ }^{1}$.

Alzheimer's disease (AD) is a degenerative brain disease and the most common cause of dementia among elderly ${ }^{2}$. According to the 2015 dementia-related statistics, published by the Alzheimer's Disease International, an estimated 46.8 million people worldwide are living with dementia, and this number will almost double every 20 years to reach 75 million by 2030 . Extensive research efforts were undertaken to improve the status and management of $A D$, despite the ethical challenges that it holds ${ }^{2}$. Moreover, these ethical dilemmas are magnified when the research is invasive, burdensome, and with unexpectable risks ${ }^{2,3}$.

"Respect for Persons" is one of the principles defined by the report of Belmont in 1979, for biomedical research. This principle identifies three elements to validate the informed consent (IC) process: (1) disclose sufficient relevant information for decisionmaking, (2) patient's comprehension, and (3) voluntariness ${ }^{4}$. Voluntariness means that the patient should take a decision without being subjected to coercion or undue influence ${ }^{4}$. This reflects the principle of autonomy which is defined in the literature as "a person's ability and opportunity to make decisions relating to his/her own wishes" ${ }^{5}$, or as "an act of self-determination exercised by a competent person" ${ }^{3}$. In a democratic society, many independent people are autonomous, but not all people with autonomy are necessarily independent. A person who is bound to a wheelchair can still exercise the power to order someone to carry his wishes. The appearance of dependency and its connotation are often misinterpreted as a loss of autonomy, and this can lead to unethical behavior ${ }^{5}$.

On the other hand, comprehension concept of the IC process, require that the person understand adequately the information provided ${ }^{4}$, and has the capacity to make the decision in question ${ }^{6}$. Capacity evaluation is a two-step process, where the clinician starts by assessing a person's decisional abilities, followed by a judgment regarding the patient's capacity to make a particular decision (e.g., consent) ${ }^{7}$. Decision-making capacity (DMC) must be assessed in relation to the particular decision a patient needs to make at the time the decision needs to be made ${ }^{7}$. A common dilemma that might emerge during the management process, occur during the DMC testing, to either consent to treatment or participate in research trials ${ }^{8}$.

When a clinician makes a judgment of impaired capacity, his decision should rely on the severity of the cognitive impairment, context, decision that needs to be taken, and risk-benefit ratio of the various options ${ }^{7}$. Hughes $(2001)^{9}$ stated that "it is important to see the person as one who is acting and interacting in a cultural-historical context in which he/she is embedded". This could imply 
that even if the people suffer from dementia, they are not automatically detached from their historical origin and, until they reach a vegetative state of total unawareness of their surroundings, they should be considered as retaining some extent of autonomy ${ }^{5}$. Moreover, Hegde et al. (2016) ${ }^{7}$ stated that capacity, though dependent on cognition, is not the same as cognition. Moreover, both of cognitive function and decisional ability might vary with time for any individual. Therefore, clinicians must take notable considerations to understand the relationship between these two parameters as it has a significant impact on their judgment of $\mathrm{DMC}^{7}$.

For instance, high scores on Mini-Mental State Examination (MMSE) may indicate better decision-making ability, however, a normal MMSE does not rule out impaired capacity, and i would be preferable to use the MMSE in conjunction with other tests to improve the patient's comprehension of the tasks ${ }^{7}$. On the other hand, a promising alternative to the MMSE due to its high sensitivity to early detection of dementia and mild cognitive impairment $(\mathrm{MCl})$ is the Montreal Cognitive Assessment (MoCA) scale ${ }^{10}$. MoCa is a rapid screening instrument for mild cognitive dysfunction. MoCA evaluates different types of cognitive abilities and showed to outperformed the MMSE among both $\mathrm{MCl}$ and $\mathrm{AD}$ with high sensitivity $(1.00)^{11}$. However, specificity was observed to be lower than that of the MMSE although still high $(0.87)^{11}$. Moreover, when a cut score of 23 was used, both sensitivity and specificity were excellent (0.96 and 0.95 , respectively $)^{12 .}$

It is noteworthy that there is currently no single test, that could be considered as the gold standard for capacity assessment ${ }^{2}$. MacArthur Competency Assessment Tool (MacCAT) is currently the most used and the best validated semi-structured interview that assesses a person's capacity to choose, understand, appreciate, and reason ${ }^{2}$. However, it requires adequate training to use and is time-consuming ${ }^{13 .}$ Moreover, the validity of the final judgment of DMC depends on the use of other clinical variables and clinician values since the MoCa does not establish a cutoff for capacity ${ }^{14}$. On the other hand, a more recent tool, the University of California, San Diego Brief Assessment of Capacity to Consent (UBACC), seems interesting for routine practice due to its simplicity, relevance, and applicability in older patients ${ }^{13}$. Yet, this tool does not evaluate the capacity to express a choice. The UBACC is a potentially useful instrument for screening to identify those who are in need of more comprehensive decisional capacity assessment, and not to come up with final judgments ${ }^{15}$. Moreover, many clinicians or researchers use solely their clinical experience to judge patients' DMC, however, this approach is imprecise ${ }^{13}$.

In practice, a combination of clinician's judgment with a structured capacity interview and neuropsychological tests that include executive function tests, that assess decisional ability would be ideal ${ }^{7}$.

On the other hand, as the disease progresses, most people with dementia lose their capacity to make some important decisions, so potential friends, family, caregivers or other proxies are often involved in DM and advocating for their wishes ${ }^{16}$.

To our knowledge, the issue of IC and the principle of autonomy among the geriatric population is not yet studied in the Lebanese context. Therefore, the primary objective is to investigate the Geriatricians' standpoint regarding the DMC of their patients with $A D$, regarding the consent for research trial and/or therapeutic participation. The secondary objective is to propose an algorithm for a reliable, comprehensive, and valid assessment of the DMC.

\section{Methods}

\section{Design}

In order to conduct the study and describe the standpoint of the Geriatrician regarding DMC of their patients with AD, a crosssectional descriptive e-survey was designed. Moreover, ethical approval was acquired from the Lebanese University institutional review board (IRB). All the data collected were confidential and reviewed only by the study principal investigators. 


\section{Participants and selection criteria}

Lebanese Geriatricians formed the population of interest for this study. Moreover, it is noteworthy to mention that there is a major deficiency in the number of physicians specialized in Geriatrics in Lebanon ${ }^{17}$. Participants who did not work with patients having $A D$, either clinically or on a research basis, and practicing outside of the Lebanese territories, constituted the exclusion criteria (Figure 1).

\section{Procedure}

Google Forms platform was utilized to create and carry on this e-survey and collect data. A list of the data that belong to the Geriatricians, who are members of the Lebanese Society for Geriatric Medicine was requested and obtained from the Lebanese Order of Physician-Beirut.

Afterward, each participant was contacted via a phone call, to explain the study purpose, and send them thereafter the survey link. Working with patients having $A D$ was a filter question to proceed with the Geriatrician. Reminders phone calls were conducted twice a week, for a period of one month.

Moreover, Geriatricians' IC was obtained systematically once they have read both the "Introduction" and "Aim" sections and accepted to fill in the remaining sections. It is noteworthy, that Geriatricians' participation was fully voluntary and they had the liberty to withdraw from the survey whenever they wanted to, simply by refraining from submitting their answers.

The survey started with a brief introduction to the study, where it described the current situation regarding aging and the increased prevalence of dementia among the elderly. It also drew the attention regarding the ethical issues that might arise during the management of these patients or while including them as potential participants to research studies. The aim of the study followed. The remaining of the survey was divided into three sections (a) section one, socio-demographic data; (b) section two, autonomy and IC; (c) section three, DMC.

\section{Section one: socio-demographic data.}

This section was dedicated for the collection of the socio-demographic data related to the Geriatricians, such as their years of experience, their gender, and their area(s) of practice [(a) academic, (b) research, (c) clinical, (d) administrative, or (e) consultation]. The last question was related to the clinical/research practice setting (s) [(a) university-hospital, (b) private clinic, (c) or specialized geriatric center].

\section{Section two: autonomy \& informed consent.}

Geriatricians' knowledge related to both the concept of autonomy and to the process of IC was tested in this section (Table 1).

\section{Section three: decision-making capacity.}

A clear distinction between both of the concepts of capacity and competency was offered at the beginning of this section. The term "capacity" refers to "a person's performance on measures of decision-making abilities" ${ }^{2}$. In addition, capacity was also defined as

"a person's ability to make a particular decision at a specific time or in a specific situation"7. The medical concept of capacity is universal 7. On the other hand, the term "competency" was defined by "the judgment that a person's capacity is adequate to make the decision in question" ${ }^{2}$, and by "legal capacity that is determined by a judge in court, where it is a threshold requirement imposed by society for an individual to retain decision-making power in a particular activity or set of activities". The judicial concept of competency is restricted by the rules of the national legal system, which will differ from country to country ${ }^{7}$. 
Afterward, three questions were designed to investigate Geriatricians' standpoint regarding the process of DMC (Table 2). If the Geriatrician answered by "No" on the first question, he/she were systematically directed to submit their answers.

\section{Statistical Analysis}

Data were analyzed using the Statistical Package for the Social Sciences (SPSS) version 21.0 for Windows. Descriptive statistics (mean, standard deviation, proportions) were calculated to characterize geriatricians' socio-demographic data and test results.

\section{Results}

Nine Geriatricians with a mean of $14.7 \pm 9.6$ years of experience completed the survey and submitted their answers. All the participants were clinicians, whereas, only $66.7 \%$ were involved in the field of research (Figure 2), and $88.9 \%$ worked in the university-hospital settings (Figure 3). Moreover, it's important to state that all of the physicians who participated in this study had an initial specialization in fields other than the Geriatrics (internal medicine, family medicine, endocrinology, and metabolism), then completed their training in gerontology, and eventually joined the Lebanese Society for Geriatric Medicine.

Only $33.3 \%$ of the Geriatrician were able to recognize correctly the statements that reflect the principle of autonomy. Whereas, all the participants agreed that the process of acquiring the IC of the patients with $A D$ to be a must in the research or clinical settings. However, no one recognized all the necessary elements required by the process of IC, and only $66.7 \%$ knew that a legal text exists in Lebanon regarding the process of IC.

$88.9 \%$ of the participants stated that they tested for DMC among their patients with AD. Moreover, among those who tested for the DMC used different modalities (Table 3), and had different consideration of determining whether or not their patients with AD had adequate capacity. Among the participants who had chosen the MMSE test, they had selected different cutoffs to judge their patient as having the adequate DMC (score above 20; 24; and 25). The remaining Geriatricians didn't have the ability to clearly justify their judgment.

\section{Discussion}

Within reason, it is every adult legal right to make decisions related to his or her own wishes. Incorporated in this right is the freedom to make a healthcare-related decision. Moreover, a democratic society does provide moral, ethical, and social guidelines within which these decisions must fall, in order to optimize personal freedom and autonomy, while ensuring that individual choices are within a safe, acceptable behavior and practice. The imposition of limits is a complicated issue, particularly when it comes to health-related care where a patient must demonstrate the capacity to make the decision in question ${ }^{18}$.

The findings of this study revealed that only $33.3 \%$ of the Geriatricians were able to identify correctly the statements that reflect the principle of autonomy. While the remaining Geriatricians didn't recognize that in case of emergency and when the patient's decisionmaking incapacity was confirmed, decision-making is done by a surrogate defined by a "person of trust" that reflect patient's own wishes. This was similar to a study conducted by Tarabey et al. (2018) ${ }^{19}$, where Psychiatrists showed a poor understanding of the concept of autonomy.

All Geriatricians agreed on the need of IC, among the patients with AD, in both research or clinical settings. However, no one was able to indicate all the necessary elements required by the process of IC. This is supported by the findings of a Lebanese national survey conducted by Abou-Mrad (2008) ${ }^{20}$, who highlighted the absence of consent release in around $83 \%$ of Lebanese hospitals. It's noteworthy that only one participant knew that disclosing adequate information to patients, is directly linked to the process of IC. Appelbaum (2007) ${ }^{14}$ has stated that physicians must prove good communication skills in ways that patients understand, and not just tell patients about a proposed procedure or therapy and its risks and benefits. Finally, $66.7 \%$ of the Geriatriciansnknew that a legal text exists in Lebanon regarding the process of IC (Law 574/2004, patients' rights and informed consent) ${ }^{21}$.

Furthermore, $66.67 \%$ of the Geriatricians failed to recognize that "a confirmation of a patient's competency to take a decision", is required by the process to IC. Yet, $88.9 \%$ stated that they test for DMC among their patients with AD. This reveals a mismatch 
between the rationale behind testing the $\mathrm{DMC}$ and the act of performing it.

Testing of the DMC is used to determine the extent of an individual's independence ${ }^{13}$. Therefore, making judgments based on only one parameter would be erroneous ${ }^{13}$. However, findings showed that the Geriatrician relied only on one method while testing the DMC, either a tool or clinical experience. For instance, some had chosen the MMSE and had selected different cutoffs (score above 20; 24 and 25) to judge that their patients' adequate capacity. Appelbaum (2007) ${ }^{14}$ stated that when scores of less than 19 were presented, the MMSE has been found to correlate with clinical judgments of incapacity. However, studies vary in suggesting that scores of 23 to 26 or higher are strongly indicative of competence ${ }^{14}$. On the other hand, Geriatricians were not able to make a clear judgment regarding their patients' capacity when they have selected either of MacCAT-CR/MacCAT-T, UBACC, or their clinical experience solely as a method to screen for the DMC. This is demonstrated by the fact that the MacCAT tool does not establish a cutoff for capacity, the validity of the final judgment will depend on other clinical variables combined with the clinician judgment ${ }^{14}$. Moreover, UBACC is potentially useful for screening purposes, and not to come up with final judgments ${ }^{15}$. Similarly, the sole use of clinical expertise to make a judgment of the patients' DMC was shown to be imprecise ${ }^{13}$.

The finding of this study motivated us to propose a set of recommendation to improve and standardize the DMC testing. We believe that the rigor of the capacity testing process should vary in relation to the severity of the likely risks and benefits of patients' decision. In practice, this involves subjecting patients who are facing more serious procedures and therapies, to more rigorous competence testing (Table 4). Strict guidelines may promote justice through reliability, yet can impede the clinician's ability to tailor adequate care to patient's personal circumstances, values, and medical history. Whereas adopting policies that are too flexible, that rely solely on the clinician experience and personal judgment, tend to compromise on reliability and with it the ethics of justice ${ }^{6}$.

While potentially helpful, tests are limited by how to be administered more consistently. For this reason, in order to reduce variability in practice, healthcare professionals must know how to use the tests properly. Furthermore, effort should be invested to help patients understand, through the use of appropriate language. Moreover, considerations should be taken regarding the educational level, cultural factors, and early identification and treatment of capacity-impairing psychiatric and medical illnesses ${ }^{6}$

Strategies to alleviate patients' fears and anxieties when it interferes with a patient's ability to attend to and process information, by introducing a known and trusted confidant or adviser to the consent process, may permit the patient to make competent judgments ${ }^{14}$. When possible, it is suggested to evaluate the patients several times before labeling them as incompetent ${ }^{18}$. However, if despite such efforts, the patient's incapacity to make a decision continues to persist, a proxy must be sought ${ }^{14}$. Moreover, in emergencies, physicians can provide appropriate care under the presumption that a reasonable person would have consented to such treatment ${ }^{14}$.

Moreover, substantive critics take place when the judgment relies exclusively on patient cognitive processes, while the patients' values and emotion are discarded since emotions often provide a valid reason for action ${ }^{22}$. Therefore, it is necessary that a valid DMC assessment includes an examination of the moral relevance of patient values ${ }^{6}$.

Regarding the cognitive testing, MoCA scale is a promising alternative to the MMSE due to its high sensitivity in the early detection of $A D$ and $\mathrm{MCl}^{10,11}$. For what relates to capacity testing, it's noteworthy that the "Understanding" capacity is affected the earliest, followed by reasoning and appreciation while making a "choice" is preserved the longest ${ }^{13}$. Furthermore, when a simplified threeitem questionnaire (cutoff of 2.5), was compared to MacCAT-CR to test the "Understanding subscale", it achieved a sensitivity of $100 \%$ and specificity of $77.3 \%$. This finding suggests that a reasonable alternative for MacCAT-CR might be to use this shorter tool, allowing researchers to screen for those who are in need of comprehensive capacity evaluation ${ }^{23}$.

\section{Conclusions}

The current study showed that Lebanese Geriatricians had a poor understanding of both the concept of autonomy and the criteria needed for an adequate IC process. Moreover, they were unable to select adequate tools to assess and judge the DMC of their patients with $A D$. 
It is the clinician's duty to ensure that contextual and personal factors are taken into consideration, to enable the patients to make appropriate decisions. This includes spending time to educate people, their families, ensure that patients adequately understand the disclosed information, alleviate patient's anxieties, taking into account lucid intervals, financial coverage ${ }^{9}$, and any disease that may interfere with DMC.

In this paper, we are proposing a comprehensive, logical, and valid way to improve and standardize the process of DMC testing. We believe that this could be achieved through adopting policies that attempt to balance objective criteria with clinical judgment. Moreover, we believe that the rigor of the capacity testing process should vary in relation to the seriousness of the procedures and therapies.

Cognitive status testing comes first by the mean of neuropsychological tests that include executive function tests (MoCA scale, a cut-off score of 23). Whereas, capacity testing may start by screening patients' understanding. This could be achieved by using the three-item questionnaire (cut-point of 2.5). In the case of impairment, testing can be proceeded by a comprehensive structured capacity interview "MacCAT". This testing algorithm is combined with the clinician's ability to individualize care.

Introducing a known and trusted confidant or adviser to the consent process, may permit the patient to make competent judgments when their anxieties interfere with their ability to attend to and process information. A proxy must be sought when the patient's incapacity to make a decision continue to persist. Finally, in case of an emergency, the physician can provide care, and make appropriate decisions on behalf of the patient.

\section{List Of Abbreviations}

Alzheimer's disease (AD)

Decision-making capacity (DMC)

Informed consent (IC)

Mini-Mental State Examination (MMSE)

Mild cognitive impairment (MCl)

Montreal Cognitive Assessment (MoCA)

MacArthur Competency Assessment Tool (MacCAT)

University of California, San Diego Brief Assessment of Capacity to Consent (UBACC)

Institutional review board (IRB)

\section{Declarations}

\section{Ethics Approval and Consent to Participate}

Ethical approval was acquired from the Lebanese University institutional review board (IRB). Moreover, the consent obtained from study participants was written.

\section{Consent for Publication}

Not applicable

\section{Availability of Data and Material}

The datasets used and/or analyzed during the current study are available from the corresponding author on reasonable request.

\section{Competing Interests}

Page $7 / 12$ 
The author declare that he has no competing interests.

Funding

Not applicable,

\section{Authors' Contributions}

FMA, conceptualized and designed this study, collected the data and their management; FMA contributed to the statistical analyses; FMA, analyzed and interpreted the data; FMA, drafted the manuscript and made the final approval of the version to be published.

\section{Acknowledgements}

I would like to thank the Lebanese Society for Geriatric Medicine, presided by Dr. Nazem K. Bassil, for the support provided regarding the data related to Geriatricians.

\section{References}

1. Fakhro, M. A. (2018). What's really happening among the elderly population? Gerontology \& Geriatrics Studies, 3(3). doi: 10.31031/ggs.2018.03.000563.

2. Karlawish, J. H. T., Casarett,D., Propert,K.j., James, B. D., \& Clark,C. M. (2002). Relationship between Alzheimer's Disease Severity and Patient Participation in Decisions about Their Medical C are. J Geriatr Psychiatry Neurol, 15, 68-72. Retrieved from https://journals.sagepub.com/home/jgp

3. Kim, S. Y. H. (2012). The ethics of informed consent in Alzheimer disease research. Nat Rev Neurol. 7(7), 410-414. doi:10.1038/nrneurol.2011.76

4. Koonrungsesomboon, N., Laothavorn, J., \& Karbwang, J. (2015). Understanding of Essential Elements Required in Informed Consent Form among Researchers and Institutional Review Board Members. Tropical Medicine and Health, 43(2), 117-122. https://doi.org/10.2149/tmh.2014-36

5. Rosin, A. J., \& Dijk, Y. V. (2005). Subtle ethical dilemmas in geriatric management and clinical research. J Med Ethics, 31, 355359. doi: 10.1136/jme.2004.008532

6. Siegel, A. M., Barnwell, A. S., \& Sisti, D. A. (2014). Assessing Decision-Making Capacity: A Primer for the Development of Hospital Practice Guidelines. HEC Forum, 26(2), 159-168. https://doi.org/10.1007/s10730-014-9234-8

7. Hegde, S., \& Ellajosyula, R. (2016). Capacity issues and decision-making in dementia. Annals of Indian Academy of Neurology, 19(5), 34. https://doi.org/10.4103/0972-2327.192890

8. Hirschman, K. B., Joyce, C. M., James, B. D., Xie, S. X., Karlawish, J. H. T. (2005). Do Alzheimer's Disease Patients Want to Participate in a Treatment Decision, and Would Their Caregivers Let Them? The Gerontologist, 45(3), 381-388

9. Hughes, J. C. (2001) Views of the person with dementia Journal of Medical Ethics, 27, 86-91. Retrieved from https://jme.bmj.com/content/27/2/86.info

10. Mast, B. T., \& Gerstenecker, A. (2010). Screening Instruments and Brief Batteries for Dementia. InHandbook of Assessment in Clinical Gerontology (2nd Edition ed., pp. 503-530). Elsevier. doi:https://doi.org/10.1016/C2009-0-01911-4

11. Luis, C. A., Keegan, A. P., \& Mullan, M. (2009). Cross validation of the Montreal Cognitive Assessment in community dwelling older adults residing in the Southeastern US. International Journal of Geriatric Psychiatry, 24(2), 197-201. https://doi.org/10.1002/gps.2101

12. Nasreddine, Z. S., Phillips, N. A., Bacdirian, V., Charbonneau, S., Whitehead, V., Collin, I., ... Chertkow, H. (2005). The Montreal Cognitive Assessment, MoCA: A Brief Screening Tool For Mild Cognitive Impairment: MOCA: A BRIEF SCREENING TOOL FOR MCl. Journal of the American Geriatrics Society, 53(4), 695-699. https://doi.org/10.1111/j.1532-5415.2005.53221.x 
13. Gilbert, T., Bosquet, A., Thomas-Antérion, C., Bonnefoy, M., \& Le Saux, O. (2017). Assessing capacity to consent for research in cognitively impaired older patients. Clinical Interventions in Aging, 12, 1553-1563. https://doi.org/10.2147/CIA.S141905

14. Appelbaum, P. S. (2007). Assessment of Patients' Competence to Consent to Treatment. N Englj Med, 7.

15. Jeste, D. V., Palmer, B. W., Appelbaum, P. S., Golshan, S., Glorioso, D., Dunn, L. B., ... Kraemer, H. C. (2007). A New Brief Instrument for Assessing Decisional Capacity for Clinical Research. Archives of General Psychiatry, 64(8), 966. https://doi.org/10.1001/archpsyc.64.8.966

16. Lord, K., Livingston, G., \& Cooper, C. (2015). A systematic review of barriers and facilitators to and interventions for proxy decision-making by family carers of people with dementia. International Psychogeriatrics, 27 (8), 1301-1312. doi:10.1017/S1041610215000411

17. Abyad, A. (1995). Geriatrics in Lebanon: the beginning. INT'L. J. AGING AND HUMAN DEVELOPMENT, 41(4), 299-309. doi: 10.2190/AHV3-NYM3-T6CR-R3ML

18. Epstein, J. (2008). How Reliable is the Competency Assessment Process?. Virtual Mentor, 10(8),511-515. doi: 10.1001/virtualmentor.2008.10.8.jdsc1-0808.

19. Tarabey, L., El Bitar, S., Karam, B., Zaidan, J., Hayek, M., \& Abou-Mrad, F. (2018).Informed consent for shared decision-making in Lebanese psychiatric care. Ethics, Medicine and Public Health,5, 94-98. Retrieve from https://doi.org/10.1016/j.jemep.2018.03.002

20. Abou-Mrad, F. (2008) Clinical trials in Lebanon. Dissertation, Université' Paris Descartes.http://www.ethique.inserm.fr/inserm/ethique.nsf/AllDocumentsByUNID/51EDD9BA4BAEC3F5C1257718002DB0B8/

21. (2019). Ccnleorglb. Retrieved, from http://ccnle.org.lb/sites/default/files/pages/files/law_no_574_of_2004_on_patients_rightsand_informed_consent_0.pdf

22. Charland, L. C. (2011). Decision-Making Capacity. Retrieved from https://plato.stanford.edu/entries/decision-capacity/

23. Palmer, B. W., Dunn, L. B., Appelbaum, P. S., Mudaliar, S., Thal, L., Henry, R., ... Jeste, D. V. (2005). Assessment of Capacity to Consent to Research Among Older Persons With Schizophrenia, Alzheimer Disease, or Diabetes Mellitus: Comparison of a 3Item Questionnaire With a Comprehensive Standardized Capacity Instrument. Archives of General Psychiatry, $62(7), 726$. https://doi.org/10.1001/archpsyc.62.7.726

\section{Tables}

Table 1 Questions related to section two: autonomy \& informed consent. 


\section{Question 1 Which among the following statement(s) reflect on the principle of "Autonomy"?}

ə An act of self-determination exercised by a competent person. An ability and opportunity to make decisions related to the patient's own wishes.

$\partial \quad$ In the case of confirmed incompetence, and no emergency, the decision is taken by a legal surrogate defined by a "person of trust" that reflect the patient's wishes.

ð In the case of confirmed incompetence, and no emergency, the decision is taken by the treating Physician.

¿ None of the above.

Question 2 Is the process of "Informed Consent" of patients with AD, considered a must in the research or clinical settings?

i Yes

i No

Question 3 Does a Legal text exist in Lebanon regarding the process of "Informed Consent"?

i Yes

i No

Question 4 Select the necessary element(s) as required by the process of "Informed consent".

i Adequate disclosure of information.

i A context free of undue influence, allowing the patient to make a voluntary choice.

i Confirmation of a patient's competency to take a decision.

le 2 Questions related to section three: decision-making capacity.

Question 1 Do you test for decision-making capacity (DMC) among your patients with AD?

i Yes

i No

Question 2 How do you measure the DMC of your patients with AD?

i Mini-mental state examination (MMSE)

i MacArthur Competency Assessment Tool for clinical research or Treatment respectively (MacCAT-CR or MacCAT-T)

i Other (narrative answer)

Question 3 How do you determine if your patient with AD has an adequate decision-making ability based on the above scores of the DMC testing?

i Narrative answer

Table 3. Representation of the decision-making tools used by the Geriatricians in percentage. 


\begin{tabular}{|l|c|}
\hline \multicolumn{1}{|c|}{ Tool } & Percentage (\%) \\
\hline UBACC & 25 \\
\hline MacCAT-T & 12.5 \\
\hline MMSE & 37.5 \\
\hline Clinical judgment & 25 \\
\hline
\end{tabular}

Table 4. Sliding scale approach grading. Modified from Drane (1985).

\begin{tabular}{llll}
\hline & \multicolumn{1}{c}{ Decisions } & Competency \\
\hline Standard 1 & 1.Consent where risk/benefit balance is favorable & \\
\cline { 2 - 2 } & 2.Refusal where risk/benefit balance is unfavorable & LOW \\
\cline { 2 - 2 } Standard 2 & Consent or refusal where risk/benefit balance is unclear & MODERATE \\
\cline { 2 - 2 } Standard 3 & 1.Acceptance where risk/benefit balance is unfavorable & \\
\cline { 2 - 2 } & 2.Refusal where risk/benefit balance is favorable & HIGH \\
\hline
\end{tabular}

\section{Figures}
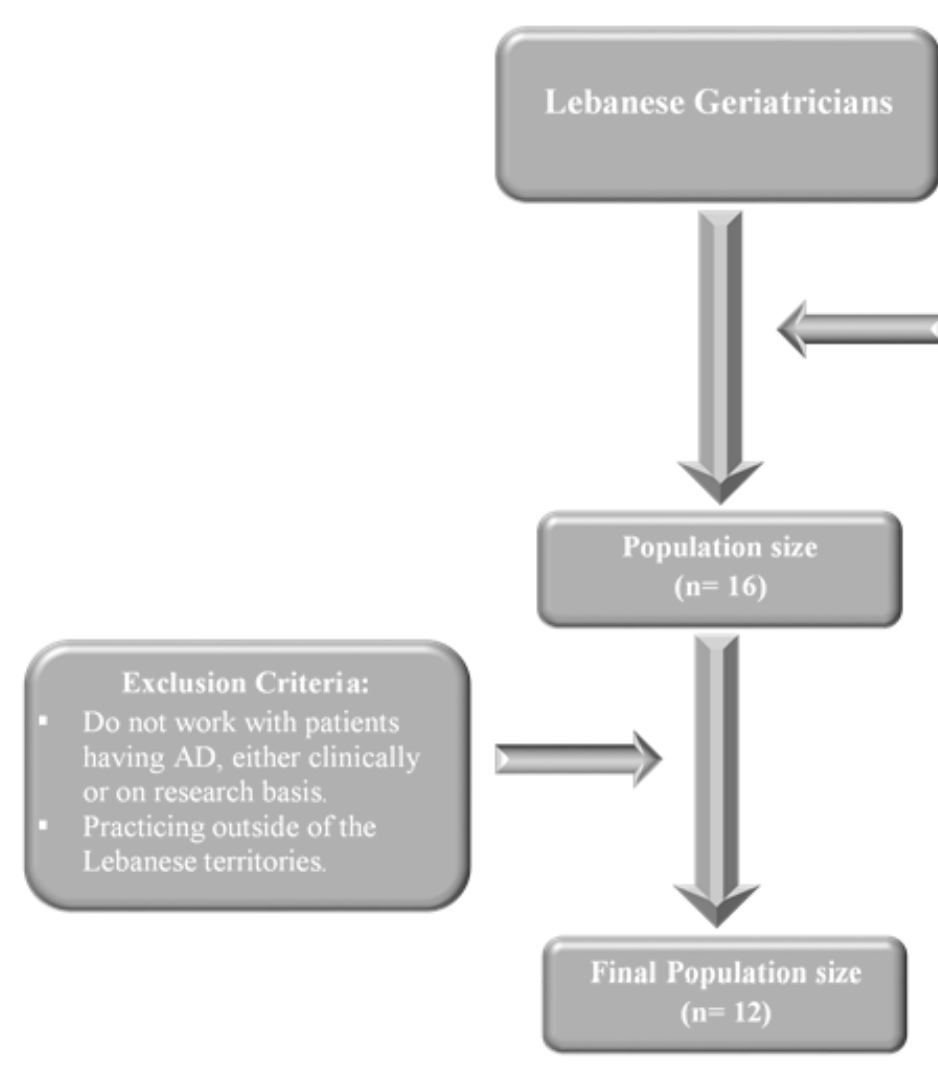

Inclusion Criterion:

Member of Lebanese Society for

Geriatric Medicine.

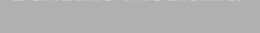




\section{Figure 1}

Flowchart representing the recruitment pool.

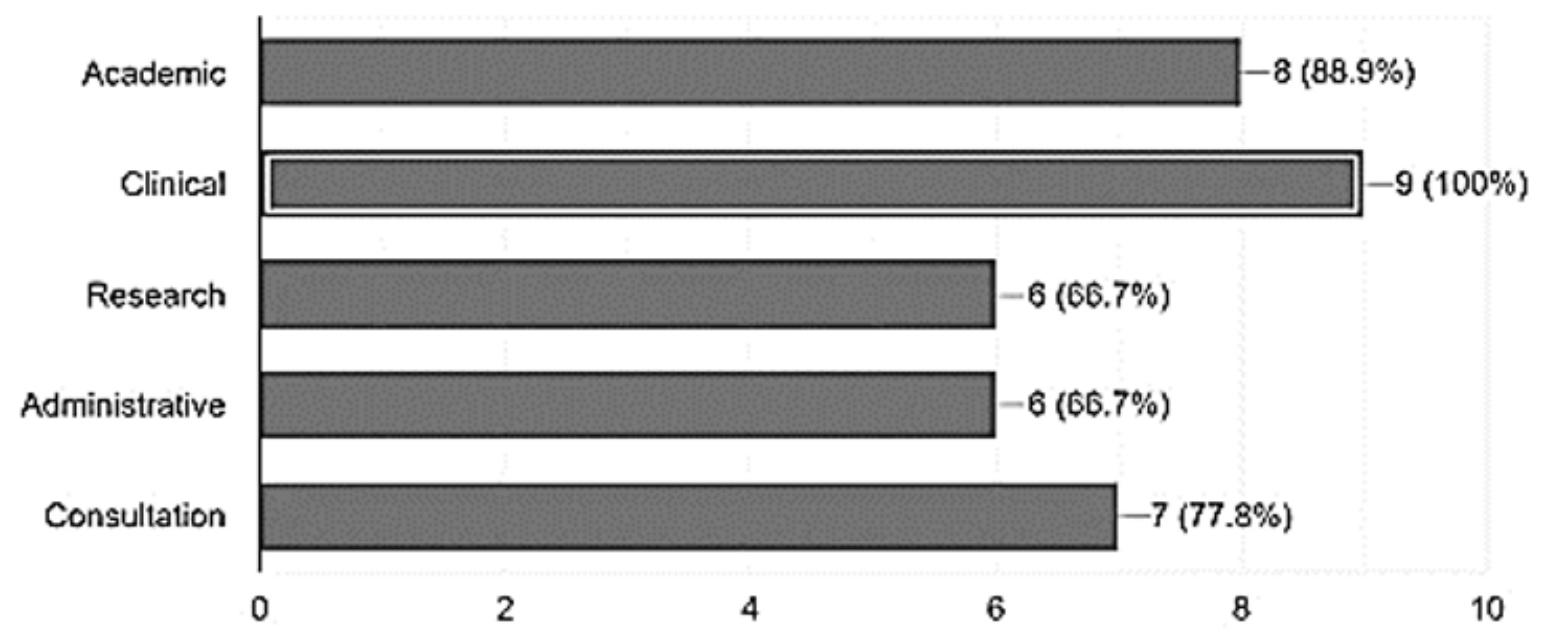

Figure 2

Distribution of the Geriatrician according to the practice area.

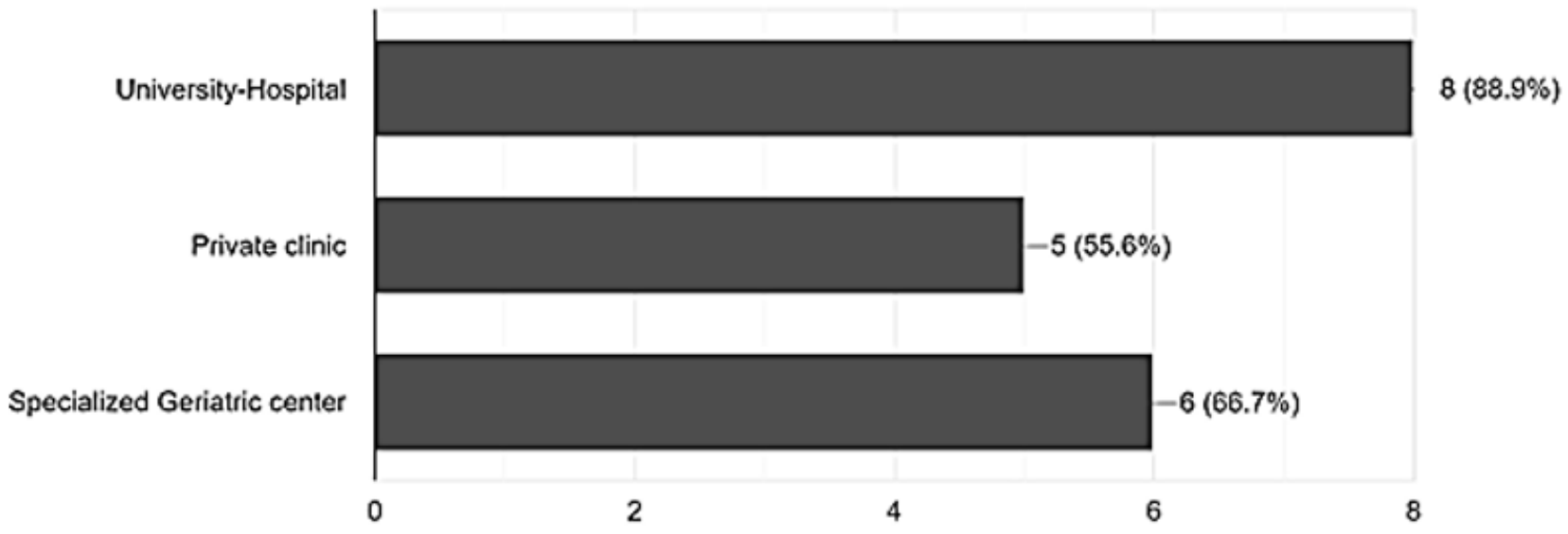

Figure 3

Distribution of the Geriatrician according to the clinical/research practice setting. 